\title{
Tratamiento con ultrafiltración lenta continua en la insuficiencia cardíaca resistente a diuréticos
}

\author{
Eider Barrena Solaguren, Lucia Setien Barreras, Horten Huerga Molinuevo, Mónica Garrido Barrientos,
} Carmen Cuevas Cabrera

Hospital de Galdakao-Usansolo. Bizkaia

\section{Introducción:}

La insuficiencia cardiaca congestiva (ICC) conlleva una sobrecarga de volumen que puede dar lugar a la aparición de un síndrome de congestión pulmonar, con disnea, derrame pleural, edema agudo de pulmón, etc, que suele acompañarse de signos y síntomas sistémicos, como ascitis, edemas,... El tratamiento de elección son los diuréticos, pero hay pacientes que no responden a ellos. En estos casos la ultrafiltración lenta continua se presenta como una opción terapéutica. Mediante esta técnica, se extrae agua plasmática de la sangre del paciente sin modificaciones electrolíticas y sin alterar el volumen sanguíneo circulante, manteniendo la estabilidad hemodinámica y mejorando la sintomatología.

\section{Objetivos:}

1. Evaluar la complejidad en la preparación y manipulación de la técnica.

2. Hacer una valoración sobre la tolerancia del paciente a la técnica.

3. Detectar posibles complicaciones: coagulación del filtro, hemorragias,...

4. Observar la mejoría de la sintomatología (disnea, edemas...)

5. Evaluar la sobrecarga de volumen con BCM.

\section{Material y método:}

Estudio retrospectivo, descriptivo y observacional con una muestra de tres pacientes usando un monitor Dedyca con su kit de SCUF y anticoagulación con enoxaheparina. Un paciente portador de FAVI (trasplantado renal) y dos con vía central femoral bilúmen. Se evaluó la sobrecarga hídrica mediante BCM en días alternos. Se les pesó, se realizaron extracciones analíticas y se midió la TA pre y postratamiento. El tratamiento constó de entre 2 y 8 sesiones. La técnica se realizó a QB 80-100 $\mathrm{ml} / \mathrm{min}$, la UF fue entre $100-300 \mathrm{ml} / \mathrm{h}$, el tiempo entre 5-6 h. Se hizo registro horario de todos los parámetros en gráfica específica.

\section{Resultados:}

El manejo y preparación de la técnica resultó muy sencillo y precisó poco tiempo de montaje. Para valorar la tolerancia nos basamos en registros horarios de tensión arterial, frecuencia cardíaca y mantenimiento del Hematocrito, no apreciándose cambios significativos ni variaciones hemodinámicas. Resultó bien tolerada. Los dos pacientes con catéter femoral tuvieron problemas de flujo. Además, en uno de ellos hubo que cambiar el circuito por entrada de aire. El paciente portador de FAVI no tuvo problemas con el acceso vascular, pero sí con uno de los sensores del circuito que se manchó de sangre y no permitió medir la presión arterial. En cuanto a la valoración de la disnea se comprobó en 2 pacientes que mejoraba a lo largo del tratamiento y en el otro paciente no era valorable por el estado neurológico que presentaba. Respecto a la BCM, no se pudo realizar a un paciente por ser portador de marcapasos. En los otros dos casos se verificó que tenían sobrecarga hídrica. 


\section{Conclusiones:}

Resultó ser una técnica simple de manejar, de fácil preparación y bien tolerada por los pacientes. Se ha demostrado eficaz, puesto que siendo uno de los principales objetivos la eliminación de líquido, lo hace de forma gradual y controlada, consiguiendo así la mejora de la clínica. Por todo lo expuesto, la SCUF puede considerarse una buena alternativa en pacientes con ICC refractaria al tratamiento con diuréticos.

\section{Referencias Bibliográficas}

1. Costanzo MR, Guglin ME, Saltzberg MT, Jessup $M L$, Bart BA, Teerlink JR, et al. Ultrafiltration Versus Intravenous Diuretics for Patients Hospitalized for Acute Decompensated Heart Failure. JACC. 2007; Vol. 49, No. 6.

2. Bart $B A$, Goldsmith $S R$, Lee $K L$, Givertz $M M$, O'Connor CM, Bull DA, et al. Ultrafiltration in Decompensated Heart Failure with Cardiorenal Syndrome. N Engl J Med 2012; 367: 2296-2304.

3. Patarroyo M, Wehbe $E$, Hanna M, Taylor D, Starling R, Demirgian S, et al. Cardiorenal Outcomes After Slow Continuous Ultrafiltration Therapy in Refractory Patients With Advanced Decompen- sated Heart Failure. JACC. 2012; 60(19): 19061912.

4. Jaski B, Romeo A, Ortiz B. Outcomes of volumen overloaded cardiovascular patients treated with ultrafiltration. J Cardiac Fail 2008; 14: 515-520.

5. Liang K, Williams A, Greene E, Redfield M. Acute decompensated heart failure and the cardiorenal syndrome. Crit Care med 2008; 36: 75-88.

6. Bart B, Boyle A, Bank AJ. Ultrafiltration versus usual care for hospitalized patients with heart failure: the relief for acutely fluid-overloaded patients with decompensated Congestive Heart Failure (RAPIDCHF) trial. JACC 2005; 46: 2043-2046.

7. Freda BJ, Mallidi J, Braden GL. Diuretics or Ultrafiltration for Acute Decompensated Heart Failure and cardiorenal syndrome? AJKD 2013 Mar 30; pii. S0272-6386 (13) 00567-2.

8. Guthrie RM. Review of diuretic and ultrafiltration strategies in patients with acute decompensated heart failure. Hosp Pract (1995); 2013 Feb 41 (1): 129-31.

9. Prosek J, Agarwal A, Parikh SV. Cardiorenal syndrome and the role of ultrafiltration in heart failure. Curr Heart Fail Rep. 2013 Mar 10(1): 81-8. 